\title{
Access to dimensional values can be unselective during early perceptual processing
}

\author{
STEPHEN DOPKINS \\ George Washington University, Washington, D.C.
}

\begin{abstract}
On each trial of the primary task for the study, a pair of small circles appeared on a computer screen. Across trials, the circles appeared in an array of positions on the screen. The participant was required on each trial to indicate whether or not the test circles were two or fewer position units apart in terms of the horizontal dimension of this array. The discrimination that the task required was easier to the extent that the vertical distance between the circles was ignored. When the task was performed under a high degree of speed stress, vertical distance and horizontal distance exerted equivalent effects on measures of performance. When the task was performed under a lesser degree of speed stress, horizontal distance exerted a larger effect than vertical distance did. The results supported the early holistic hypothesis over the dimensional similarity hypothesis as an account of the way information from different dimensions is accessed in perceptual processing.
\end{abstract}

Humans tend to understand the world in quantitative terms. We think of a watermelon not simply as large or small but, rather, as having a certain particular size, relative to other things that we might experience. In fact, we generally think of things as having multiple quantitative attributes. In addition to size, the watermelon will have a certain weight, age, cost, and so forth. Thus, human experience unfolds within a framework of perceptual dimensions. The multidimensional character of experience imposes demands on the human perception system. In particular, considerable flexibility is required. In order to completely understand a given stimulus, one must typically evaluate it on many dimensions. At the same time, situations arise in which one must evaluate the stimulus on just one of the many relevant dimensions.

The human capacity for evaluating a stimulus on a single perceptual dimension has been studied with two speeded classification tasks. In these tasks, participants are required to sort simple stimuli into categories on the basis of their values on perceptual dimensions. In the experimental condition of the correlated values task, the stimuli vary on a focal dimension, on which the required categorization is supposed to be based, and on another, nonfocal dimension, and the values on the two dimensions are correlated (see Figure 1). In the baseline condition, the stimuli vary only on the focal dimension. A redundancy effect is said to occur if responses are faster and/or more accurate in the experimental condition than

This research was supported by National Institute of Mental Health Grant 66189. The author thanks R. D. Melara for helpful comments regarding a previous version of this article. Correspondence concerning this article should be addressed to S. Dopkins, Psychology Department, George Washington University, 2125 G Street, NW, Washington, DC 20052 (e-mail: dopkins@gwu.edu). in the baseline condition (Garner, 1974; Garner \& Felfoldy, 1970; Melara, 1989; Melara, Marks, \& Potts, 1993b; Smith \& Kemler, 1978). In the experimental condition of the orthogonal values task, the stimuli again vary on a focal and a nonfocal dimension, but here the values on the two dimensions are uncorrelated (see Figure 2). The baseline condition is the same as that for the correlated values task. An interference effect is said to occur if responses are slower and/or less accurate in the experimental condition than in the baseline condition (Garner, 1974; Garner \& Felfoldy, 1970; Melara, 1989; Melara et al., 1993b; Smith \& Kemler, 1978). On the basis of work with the correlated values and orthogonal values tasks (as well as with other tasks), two kinds of perceptual dimensions have been distinguished. Interacting dimensions are associated with redundancy effects in the correlated values task and with interference effects in the orthogonal values task. Noninteracting dimensions are associated with neither redundancy nor interference effects.

Psychologists have been particularly intrigued by the failures of selective evaluation that occur with interacting dimensions. Until recently, the prevailing view of such failures has been the early holistic hypothesis. This hypothesis claims that when a stimulus has values on two interacting dimensions, access to these values is often completely unselective during the early stages of perceptual processing. Only during later stages of processing is access to this dimensional information selective. As a consequence, pairs of interacting dimensions are often perceptually inseparable during the early stages of processing. In other words, the perceptual effect of a stimulus on one dimension is often influenced by the value of the stimulus on the other dimension. It is for this reason that we observe redundancy effects in the correlated values task and interference effects in the orthogo- 


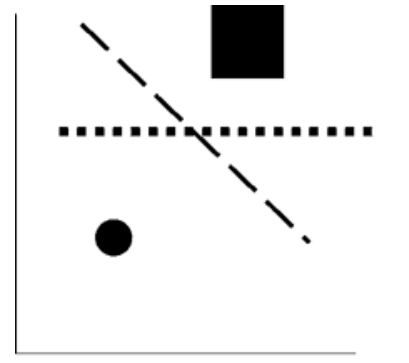

Experimental condition

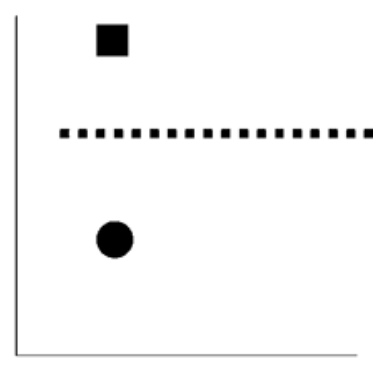

Baseline condition

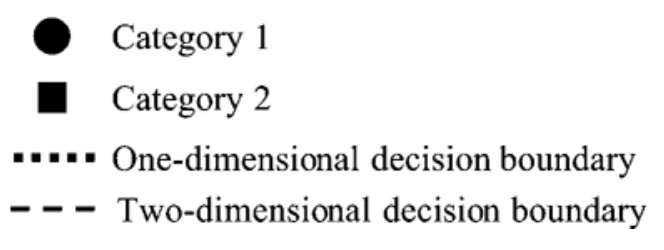

Figure 1. The correlated values task. In the experimental condition, the stimuli vary on the focal dimension, on which the required categorization is supposed to be based, and on another, nonfocal dimension, and the values on the two dimensions are correlated. In the baseline condition, the stimuli vary only on the focal dimension. With the one-dimensional rule, response decisions are based on information from the focal dimension. With the two-dimensional rule, response decisions are based on information from both dimensions of the stimulus set.

nal values task (Grau \& Kemler Nelson, 1988; Kemler Nelson, 1993; Lockhead, 1972, 1979).

Recently, the early holistic hypothesis has come under attack. Exception has been taken, in particular, to the view that access to information from interacting dimensions is completely unselective during early perceptual processing. According to the dimensional similarity hypothesis, stimuli undergo two sorts of processing when they are perceived. In attribute-level processing, which occurs for all stimuli, information is considered that independently locates a given stimulus on all of the dimensions along which it varies. In stimulus-level processing, which occurs for stimuli that vary on pairs of interacting dimensions, information is jointly considered that locates each stimulus on both of the dimensions along which it varies. Because attribute-level processing occurs for all stimuli, including stimuli that vary along interacting dimensions, it is never the case that access to information from interacting dimensions is completely unselective, even during the early stages of processing. As a consequence of stimulus-level processing, however, access may be somewhat unselective (Melara, 1992; Melara \& Marks, 1990b).

As a key part of their challenge to the early holistic hypothesis, dimensional similarity proponents have criticized the interpretation of redundancy and interference effects. In questioning the interpretation of redundancy effects, dimensional similarity proponents have appealed to a distinction between perceptual and decision processes (Maddox, 1992, 2001; Maddox \& Ashby, 1996). Early holistic proponents are interested in perceptual processes. They wish to interpret redundancy effects as evidence that the focal and the nonfocal dimensions are perceptually inseparable - that the perceptual effect of a stimulus on the focal dimension is influenced by the value of the stimulus on the nonfocal dimension. Dimensional similarity proponents have pointed out, however, that this does not necessarily follow. The influence of nonfocal information might be induced by decision factors. The correlated values task can be performed in two ways - on the basis of two decision rules. (1) In the case of the one-dimensional rule, response decisions are characterized by decisional separability - they are based solely on information from the focal dimension (the decision boundary is shown as the dotted line in Figure 1; Maddox, 1992). (2) In the case of the two-dimensional rule, response decisions are not characterized by decisional separability - they are based on information from both dimensions of the stimulus set (the decision boundary is shown as the dashed line in Figure 1). The required discrimination can be performed more easily under the two- than under the one-dimensional decision rule. To see this, note that in Figure 1, the points corresponding to the stimuli are farther from the two- than from the one-dimensional decision boundary. Thus, it makes sense to perform the task on the basis of the two-dimensional, rather than the one-dimensional, decision rule. Therefore, it might be the case that the focal and the nonfocal dimensions are perceptually separable but that nonfocal information is used in performing the task, because the task is thereby performed more easily. For this reason, redundancy effects are not good evidence of early holistic processing (Melara, Marks, \& Potts, 1993a).

In questioning the interpretation of interference effects, dimensional similarity proponents have focused on

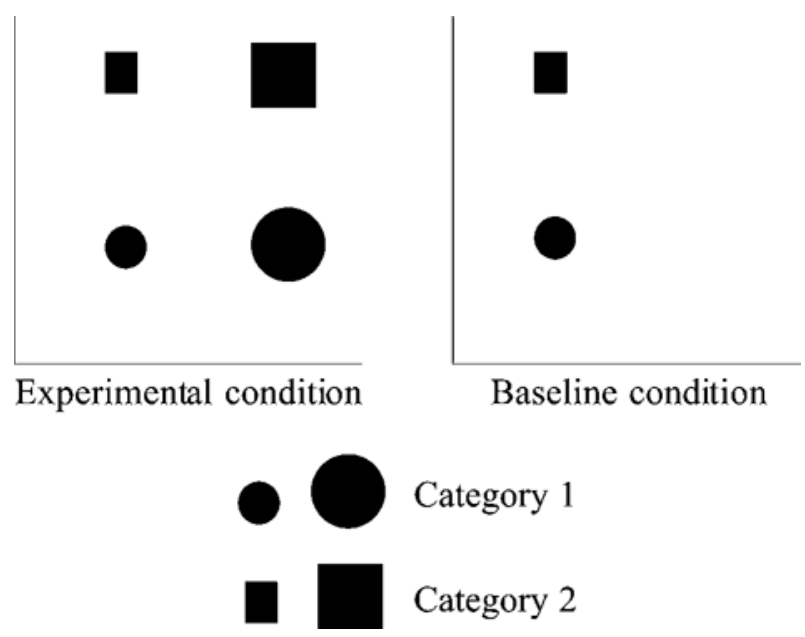

Figure 2. The orthogonal values task. In the experimental condition, the stimuli vary on the focal dimension, on which the required categorization is supposed to be based, and on another, nonfocal dimension, and the values on the two dimensions are uncorrelated. In the baseline condition, the stimuli vary only on the focal dimension. 
a crucial difference between the correlated and the orthogonal values tasks. Performance in the correlated values task improves when the stimuli vary on the nonfocal dimension. By implication, nonfocal information is used (along with focal information) in performing the task. In contrast, performance on the orthogonal values task gets worse when the stimuli vary on the nonfocal dimension. By implication, nonfocal information is not used in performing the task. Rather, such information impedes performance in some way. But nonfocal information might impede performance through many different mechanisms. Thus, interference effects constitute somewhat nonspecific evidence of the role of nonfocal information in the orthogonal values task. To drive their point home, dimensional similarity proponents have called attention to the fact that interference effects occur even when the dimensions of the stimulus set pertain to different modalities. Because it is unlikely that access to dimensional information from different modalities is completely unselective, it follows that interference effects may reflect factors other than failures of selective access. For this reason, interference effects are not good evidence of early holistic processing (Melara et al., 1993a).

The present study is an attempt to gather new support for the early holistic hypothesis and its claim that access to information from interacting dimensions can be completely unselective during the early stages of perceptual processing. In the study, this involved using a different strategy than had been used heretofore. Most of the time in each experiment was spent in a complex distance task. On each trial, a pair of small circles appeared on a computer screen. Across trials, the circles appeared in a total of 21 different positions on the screen. These positions were arranged along the horizontal and vertical dimensions of the screen in three rows and seven columns, so as to define an array such as that shown in Figure 3. The participant was required on each trial to indicate whether or not the test circles were 2 or fewer position units apart in terms of the horizontal dimension of the position array.

The complex distance task could be performed on the basis of two decision rules. (1) Under the one-dimensional rule, performance in the task was characterized by decisional separability; response decisions were based on the distance between the test circles on the focal horizontal dimension. (2) Under the two-dimensional rule, performance in the task was not characterized by decisional separability; response decisions were based on the aggregate distance between the test circles on the nonfocal

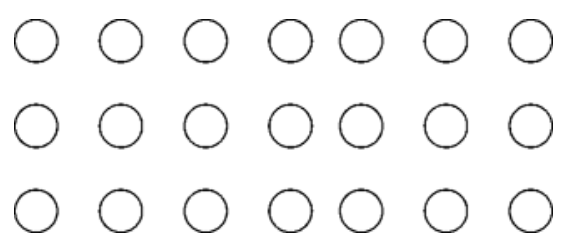

Figure 3. Position array used in the experiments of the study. vertical and the focal horizontal dimensions. The required discrimination could be performed more easily on the basis of the one- than of the two-dimensional rule. Under the two-dimensional rule, the discrimination could be performed more easily to the extent that focal distance was weighted more heavily than nonfocal distance in the aggregate distance on which response decisions were based (this is shown in more detail in the Method section). Of course, with focal distance given no weight at all, the two-dimensional rule becomes the one-dimensional rule. Figure 4 illustrates the two decision rules.

Pilot work suggests that performance of the complex distance task will be based on the two-dimensional decision rule. Both the early holistic and the dimensional similarity hypotheses accommodate this pattern. Under the early holistic hypothesis, use of the two-dimensional rule is accommodated because access to information from interacting dimensions can be completely unselective. Under the dimensional similarity hypothesis, use of the two-dimensional rule is accommodated because access to information from interacting dimensions is always somewhat unselective, because (1) stimulus-level processing always occurs for interacting dimensions and (2) stimulus-level processing does not allow information from one of two interacting dimensions to be considered independently of information from the other dimension. Thus, under both hypotheses, participants use the twodimensional rule in the complex distance task, because access to information from the interacting dimensions is at least somewhat unselective.

The question at issue in the study was exactly what sort of two-dimensional decision rule would be used in performing the complex distance task; more specifically, the question was whether focal distance and nonfocal distance would be given equal weight in the aggregate distance upon which response decisions were based. The plan was to ask this question during early and later stages of processing in the task. The goal was to show that the two dimensions were given equal weight during early processing and unequal weight during later processing. This would be used to argue that access to information from the two dimensions was completely unselective during early processing and selective during later processing, as is predicted by the early holistic hypothesis. Of course, use of a particular decision rule is not in and of itself diagnostic with respect to either of the hypotheses under consideration. Traditionally, decision questions have been seen as orthogonal to questions regarding the selectivity of access (Maddox, 1992). The goal, however, was to demonstrate a pattern of early and late decision rule usage that could not easily be explained without positing a change from unselective to selective access.

Each experiment in the study also included a simple distance task, which was the same as the complex distance task, except that only the middle row of the position array was used. The plan was to compare performance on this task with performance on trials of the 


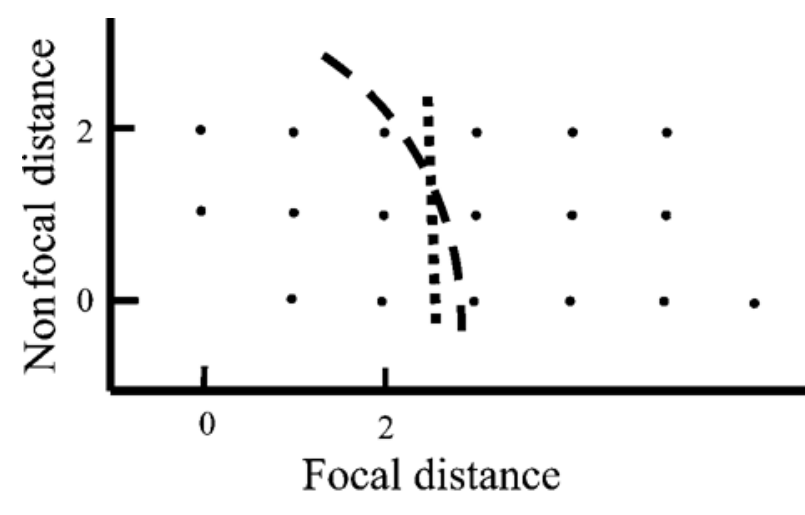

-... One-dimensional decision boundary

Figure 4. Hypothetical decision rule for the complex distance task, with focal distance and nonfocal distance given equal weight.

complex task on which the test circles did not differ on the vertical dimension. In this way, it would be possible to gauge the cost of attending to two dimensions, rather than to one.

\section{EXPERIMENT 1}

This experiment was the first step in building a case for the early holistic hypothesis. Participants performed the complex and simple distance tasks under a response signal procedure that induced speeded responding. It was assumed that the tasks would tap early perceptual processing when performed under these conditions.

The early holistic hypothesis predicted that focal and nonfocal distance might be given equal weight in the complex distance task. Although the required discrimination could be performed more easily to the extent that focal distance was given greater weight than nonfocal distance was, access to information from interacting dimensions could be completely unselective during early perceptual processing. Thus, the two dimensions might have to be given equal weight because access was unselective. In contrast, the dimensional similarity hypothesis predicted that focal distance would be given greater weight than nonfocal distance would in the complex distance task. The required discrimination could be performed more easily to the extent that this was done, and access of information from interacting dimensions would always be somewhat selective. The objective was to gather support for the early holistic hypothesis by showing that focal and nonfocal distance were given equal weight in the complex distance task.

Note that, in contrast to the case with the correlated values task, the use of nonfocal information could not have been induced in the complex distance task by decision factors. This follows because the required discrimination could be performed more easily to the extent that this information was excluded. Thus, if focal distance and nonfocal distance were given equal weight, this could not have been done in the service of a more optimal decision, as with the correlated values task. Of course, other decision factors might have caused the equal weighting of focal distance and nonfocal distance. The plan was that these other factors be dealt with in further experiments if evidence for equal weighting was observed.

\section{Method}

Participants. The participants were 18 students at George Washington University. They received extra credit in a psychology course in exchange for their efforts.

Stimuli. Each of the test circles measured $3 \mathrm{~mm}$ in diameter. Pairs of adjacent positions in the position array were separated by $8 \mathrm{~mm}$ on the horizontal and the vertical dimensions.

Decision rules for the complex distance task. Under the onedimensional rule, response decisions were based on the distance between the test circles on the focal horizontal dimension. Under the two-dimensional rule, response decisions were based on the aggregate distance between the test circles on the nonfocal vertical, as well as the focal horizontal, dimension. Although this was not the way the task was supposed to be performed, this decision rule was nonetheless capable of producing accurate results. This was shown as follows. It was assumed that focal distance and nonfocal distance would be aggregated according to the Euclidean metric. This seemed reasonable, inasmuch as the distances pertained to a space that is generally understood in Euclidean terms. It was assumed that focal distance would be given equal or greater weight, relative to nonfocal distance, in the aggregate distance on which decisions were based. Given these assumptions, simple calculation showed that, for positive trials, the aggregate distance between the test circles was always less than or equal to 2.83 position units and that, for negative trials, the aggregate distance was always greater than or equal to 3 units (see Figure 4). With focal distance given greater weight than nonfocal distance, the aggregate distance on positive trials was always less than or equal to a value that lay between 2 and 2.83 units, with the value being closer to 2 the smaller the weight given to nonfocal distance. The aggregate distance on negative trials was again always greater than or equal to 3 units.

More important, the required discrimination could be performed more easily, under the two-dimensional rule, to the extent that focal distance was given greater weight than nonfocal distance was. With focal distance and nonfocal distance given equal weight, the most difficult discrimination contrasted distances of 2.83 and 3 units. To the extent that focal distance was given greater weight than nonfocal distance was, the discrimination became easier, becoming easiest if nonfocal distance was given no weight at all.

Design. The test items for the complex distance task were created with the following three-step procedure. (1) Eighty-four items were created for which the focal/horizontal distance between the test circles was 0 . These items were created as follows. Three items were constructed for each of the seven horizontal positions in the stimulus set. The 3 items for a given horizontal position differed in the vertical positions of the test circles. Across the 3 items, the circles appeared in the three unordered pairs of vertical positions that could be formed by sampling twice, without replacement, from the three vertical positions in the stimulus set. Each of the items created in this way was repeated four times in the test sequence. (2) Two hundred sixteen and 180 items were created for which the focal/ horizontal distance between the test circles was 1 and 2, respectively. These items were created as follows. There were six and five possible unordered pairs of horizontal positions for which horizontal distance was equal to 1 and 2, respectively. For each of these pairs of horizontal positions, 9 items were constructed. The 9 items for a given pair of horizontal positions differed in the vertical positions of the test circles. Across the 9 items, the circles appeared in the 
nine ordered pairs of vertical positions that could be formed by sampling twice, with replacement, from the set of three vertical positions in the stimulus set. Each of the items created in this way was repeated four times in the test sequence. (3) Seventy-two, 54, 36, and 6 items were created for which the focal/horizontal distance between the test circles was $3,4,5$, and 6 , respectively. These items were created as follows. There were four, three, two, and one possible unordered pairs of horizontal positions for which horizontal distance was equal to $3,4,5$, and 6 , respectively. For each of these pairs of horizontal positions, 9 items were constructed. The 9 items for a given pair of horizontal positions differed in the vertical positions of the test circles. Across the 9 items, the circles appeared in the nine ordered pairs of vertical positions that could be formed by sampling twice, with replacement, from the set of three vertical positions in the stimulus set. Of the 9 items that were created by crossing the first and the seventh horizontal position, the 6 items were removed for which the test circles did not have the same vertical position. Each of the items created in this way was repeated twice in the test sequence.

In this way, 648 items were created for the complex distance task. Of these, 480 were positive items, for which the test circles were 2 or fewer position units apart on the focal/horizontal dimension, and 168 were negative items, for which the test circles were 3 or more position units apart on that dimension. The items were presented in a different random order to each participant.

The test items for the simple distance task were created as follows: There were six, five, four, three, two, and one possible unordered pairs of horizontal positions for which horizontal distance was equal to $1,2,3,4,5$, and 6 , respectively. For each of these pairs of horizontal positions, 1 item was constructed. In the item for a given pair of horizontal positions, both of the test circles had the middle vertical position. Each of the items created in this way was repeated three times in the test sequence. In this way, 63 items were created. Of these, 33 were positive items, for which the test circles were 2 or fewer position units apart on the focal/horizontal dimension, and 30 were negative items, for which the test circles were 3 or more position units apart on that dimension.

Procedure. The participant was instructed that he/she should press the "B" key of the computer keyboard to indicate a positive response if the test circles were two or fewer columns apart in terms of the position array, and the "N" key to indicate a negative response if they were not. The sequence of test trials was broken into three parts. The first and the third parts, which were of equivalent length, were devoted to the complex distance task. The second part was devoted to the simple distance task. At the beginning of the second part, a message was presented, indicating that the participant could forget about the vertical dimension during that part.

At the beginning of each trial, a "Ready" signal appeared on the computer screen. When the participant pressed the space bar of the computer, a pair of circles appeared. Four asterisks appeared at the bottom of the screen $400 \mathrm{msec}$ after the circles appeared. The participant attempted to make his/her response concurrently with the appearance of the asterisks. If the interval between the appearance of the circles and the participant's response was less than $400 \mathrm{msec}$, the message "too fast" appeared at the bottom of the screen and remained there until the participant pressed the space bar. If the interval was greater than $650 \mathrm{msec}$, the message "too slow" appeared in the same manner. At the beginning of every sixth trial, a message appeared asking the participant to press the space bar to see the position array. When the participant pressed the space bar, the array was presented; 21 circles appeared simultaneously in the positions of the array.

Because positive trials were more crucial than negative trials to the objective of the experiment, as will be explained subsequently, an attempt was made to induce good performance on the positive trials. The participant was instructed that two kinds of errors were possible in the task. Yes errors occurred when the participant erro- neously pressed the "B" key. No errors occurred when the participant erroneously pressed the "N" key. The participant was told that he/she would receive a $\$ 5$ bonus if he/she made fewer than 20 no errors. When the participant made a no error, a large red message appeared on the screen. When the participant made a yes error, a small black message appeared. In each case, the message remained on the screen until the participant pressed the space bar.

\section{Results}

The following policies were followed in all of the analyses in the present study. The first five trials of each session were classified as practice trials. The data for these trials were excluded from all analyses. The data for trials on which the response was emitted more than $750 \mathrm{msec}$ after the response signal were also excluded from all analyses. For all of the experiments in the study, these policies resulted in the elimination of less than $2 \%$ of the data.

Complex distance task. The goal was to show that focal distance and nonfocal distance were given equal weight in the aggregate distance on which response decisions were based. To that end, performance was analyzed as a function of the focal and nonfocal distance between the test circles. On the basis of pilot work, it was expected that performance would decline with increases in focal and nonfocal distance on positive trials and improve with increases in focal and nonfocal distance on negative trials. It was assumed that this would reflect the use of the two-dimensional decision rule. Under this rule, positive responses should become more difficult with increases in focal and nonfocal distance, and negative responses should become easier with increases in focal and nonfocal distance. In order to achieve the goal of the experiment, the plan was to compare the relative sizes of the focal and the nonfocal distance effects. The data for the positive trials were most important for this plan, because the test items for the positive trials varied through the same three levels of distance $(0,1$, and 2 position units) on the focal and the nonfocal dimensions. Thus, it was possible to do a matched comparison of the two distance effects.

Table 1 presents the data for the positive trials as a function of the focal and nonfocal distance between the test circles. The error rate data were of primary interest, given that the response signal procedure was intended to control response time (RT). Error rate increased with increases in focal and nonfocal distance. Because no data were collected when focal distance and nonfocal distance were both 0 , the data were not neatly accommodated by an analyses of variance (ANOVA). A regression analysis was therefore conducted to confirm the significance of the patterns that were apparent in the data. The 144 cases in the analysis were the error rates that the 18 individual participants accrued for the eight different combinations of focal distance and nonfocal distance. The predictor variables were focal distance and nonfocal distance. To estimate the variability due to participants, 17 dummy variables were included. The independent variables were all entered into the regression model simultaneously. Table 2 reports the results of the analysis. 
Table 1

Experiment 1: Performance on the Positive Trials in the Complex Distance Task as a Function of Distance Between Test Circles on the Focal and Nonfocal Dimensions

\begin{tabular}{|c|c|c|c|c|c|c|}
\hline \multirow{3}{*}{$\begin{array}{c}\text { Focal Distance } \\
\text { (in Position Units) }\end{array}$} & \multicolumn{6}{|c|}{ Nonfocal Distance (in Position Units) } \\
\hline & \multicolumn{2}{|c|}{0} & \multicolumn{2}{|c|}{1} & \multicolumn{2}{|c|}{2} \\
\hline & $M$ & $\overline{S E M}$ & $M$ & SEM & $M$ & $S E M$ \\
\hline \multicolumn{7}{|c|}{ Error Rate } \\
\hline 0 & & & .029 & .007 & .081 & .020 \\
\hline 1 & .036 & .010 & .027 & .005 & .070 & .017 \\
\hline 2 & .042 & .013 & .068 & .014 & .247 & .031 \\
\hline \multicolumn{7}{|c|}{ Response Time } \\
\hline 0 & & & 492 & 5 & 520 & 9 \\
\hline 1 & 500 & 7 & 497 & 7 & 528 & 9 \\
\hline 2 & 516 & 8 & 519 & 7 & 547 & 9 \\
\hline
\end{tabular}

Note-Response time means include the $400 \mathrm{msec}$ that elapsed before the response signal appeared.

Both focal and nonfocal distance accounted for significant amounts of the variance in the error rate data. More crucially for the object of the experiment, focal distance and nonfocal distance exerted equivalent effects on performance; the coefficients for focal and nonfocal distance did not differ (in fact, the coefficient for nonfocal distance was nominally larger than the coefficient for focal distance) $[t(143)=1.21]$ (all statistical tests were conducted against a significance value of .05).

The RT data for the positive trials were also analyzed as a function of focal and nonfocal distance (see Tables 1 and 2). The pattern was the same as that for the error rate data. Both focal and nonfocal distance accounted for significant amounts of the variance in the RT data. The coefficients for focal and nonfocal distance did not differ $[t(143)=0.60]$.

Although they were not diagnostic in the manner of the data for the positive trials, because levels of focal and nonfocal distance were not matched, the data for the negative trials were subjected to exploratory analysis. Table 3 presents these data as a function of focal and nonfocal distance. Because no data were collected when focal distance was 6 and nonfocal distance was greater than 0 , the data were again not neatly accommodated by

Table 2

Experiment 1: Regression Analysis of Performance on the Positive Trials in the Complex Distance Task as a Function of Distance Between Test Circles on the Focal and Nonfocal Dimensions

\begin{tabular}{lccccc}
\hline \multicolumn{7}{c}{ Error Rate } \\
Distance & Coefficient & $\begin{array}{c}\text { Standard } \\
\text { Error }\end{array}$ & $\begin{array}{c}\text { Standardized } \\
\text { Coefficient }\end{array}$ & $t$ Value & $p$ Value \\
\hline \multicolumn{7}{c}{ Erroc } \\
Focal & 0.050 & 0.008 & 0.406 & 6.31 & $<.001$ \\
Nonfocal & 0.062 & 0.008 & 0.507 & 7.87 & $<.001$ \\
$\quad R^{2}: .513$ & \multicolumn{7}{c}{} \\
\multicolumn{7}{c}{ Response Time } \\
Focal & 15.01 & 2.26 & 0.322 & 6.63 & $<.001$ \\
Nonfocal & 16.70 & 2.26 & 0.359 & 7.38 & $<.001$ \\
$\quad R^{2}: .723$ & & & & & \\
\hline
\end{tabular}

an ANOVA. ANOVAs were therefore conducted on the data for the trials on which focal distance was less than 6. (This approach was deemed acceptable because only 1 data point out of 10 was excluded and because the data were not as crucial as the data for the positive trials. It was not thought possible to handle the data for the positive trials in the same way. Here, a similar approach would have excluded 2 out of 8 data points.) The analyses revealed that error rate decreased with focal distance [linear trend, $\left.F(1,17)=81.09, M S_{\mathrm{e}}=0.051\right]$ and with nonfocal distance [linear trend, $F(1,17)=18.58, M S_{\mathrm{e}}=$ $0.020]$ and that the linear trends in the effects of focal and nonfocal distance interacted in the error rate data $\left[F(1,17)=19.87, M S_{\mathrm{e}}=0.016\right]$, because the effect of nonfocal distance was stronger for smaller values of focal distance.

RT decreased with focal distance [linear trend, $F(1,17)=$ 7.37, $\left.M S_{\mathrm{e}}=4,446\right]$ and with nonfocal distance [linear trend, $\left.F(1,17)=4.99, M S_{\mathrm{e}}=3,178\right]$. The effects of focal and nonfocal distance did not interact in the RT data $\left[F(4,68)=1.03, M S_{\mathrm{e}}=1,239\right]$.

Simple distance task. Table 4 presents the data from the simple distance task. For comparison, the data are also shown for the comparable trials in the complex distance task (those on which nonfocal distance was 0 ). The error rate was smaller for the simple distance task than for the comparable trials in the complex distance task $\left[F(1,17)=7.64, M S_{\mathrm{e}}=0.020\right]$. The error rate for both tasks was a quadratic function of focal distance $[F(1,17)=$ $\left.71.68, M S_{\mathrm{e}}=0.040\right]$. The effects of task type and focal distance interacted in the error rate data $[F(5,85)=7.10$, $\left.M S_{\mathrm{e}}=0.016\right]$. The basis of this interaction was the difference between the effects of task type for focal distance $=2$ and for other values of focal distance. Although the error rate was generally smaller for the simple than for the complex task, this difference was reversed for focal distance $=2\left[F(1,17)=13.24, M S_{\mathrm{e}}=0.003\right]$.

RT was shorter for the simple distance task than for comparable trials in the complex distance task $[F(1,17)=$ $\left.3.39, M S_{\mathrm{e}}=5,015\right]$. RT for both tasks was a quadratic function of focal distance $\left[F(1,17)=36.87, M S_{\mathrm{e}}=3,885\right]$. The effects of task type and focal distance interacted in the RT data $\left[F(5,85)=5.14, M S_{\mathrm{e}}=1,855\right]$. The basis of this interaction was the difference between the effects of task type for focal distance $=2$ and for other values of focal distance. Although RT was generally shorter for the simple than for the complex task, this difference was reversed for focal distance $=2\left[F(1,17)=8.51, M S_{\mathrm{e}}=703\right]$.

\section{Discussion}

The test circles were more difficult to identify as being 2 or fewer position units apart on the focal dimension the further they were from one another on the focal and nonfocal dimensions. The test circles were easier to identify as being more than 2 position units apart on the focal dimension the further they were from one another on the focal and nonfocal dimensions. By implication, the task was performed on the basis of the two-dimensional decision rule. Response decisions were based on an aggre- 
Table 3

Experiment 1: Performance on the Negative Trials in the Complex Distance Task as a Function of Distance Between Test Circles on the Focal and Nonfocal Dimensions

\begin{tabular}{|c|c|c|c|c|c|c|}
\hline \multirow{3}{*}{$\begin{array}{c}\text { Focal Distance } \\
\text { (in Position Units) }\end{array}$} & \multicolumn{6}{|c|}{ Nonfocal Distance (in Position Units) } \\
\hline & \multicolumn{2}{|c|}{0} & \multicolumn{2}{|c|}{1} & \multicolumn{2}{|c|}{2} \\
\hline & $M$ & SEM & $M$ & SEM & $M$ & SEM \\
\hline \multicolumn{7}{|c|}{ Error Rate } \\
\hline 3 & .646 & .045 & .591 & .055 & .349 & .061 \\
\hline 4 & .220 & .041 & .252 & .038 & .194 & .057 \\
\hline 5 & .141 & .049 & .160 & .040 & .101 & .049 \\
\hline 6 & .070 & .026 & & & & \\
\hline \multicolumn{7}{|c|}{ Response Time } \\
\hline 3 & 632 & 23 & 612 & 21 & 595 & 16 \\
\hline 4 & 598 & 21 & 595 & 19 & 574 & 16 \\
\hline 5 & 584 & 23 & 578 & 17 & 575 & 14 \\
\hline 6 & 548 & 13 & & & & \\
\hline
\end{tabular}

Note-Response time means include the $400 \mathrm{msec}$ that elapsed before the response signal appeared.

gate distance that reflected the focal distance and nonfocal distance between the test circles. This is really the only interpretation that is compatible with the contrasting patterns that were observed for the positive and the negative trials.

Other, less interesting interpretations of the results are untenable in light of these contrasting results. For example, we can reject an interpretation holding (1) that response decisions were based solely on focal distance, (2) that the test circles had to be aligned on the nonfocal dimension before a judgment could be made on the focal dimension, and (3) that alignment was more difficult the more distant the circles were on the nonfocal dimension. We can also reject an interpretation holding (1) that response decisions were based solely on focal distance, (2) that the values of the test circles on the nonfocal dimension were distracting, and (3) that the amount of distraction increased with increases in the nonfocal distance between the test circles. Finally, we can reject an interpretation holding that positive responses were emitted when either focal or nonfocal distance was less than or equal to 2. Under all of these interpretations, we would have expected performance on both the negative and the positive trials to decline as a function of nonfocal distance.

Further evidence for the use of a two-dimensional decision rule comes from the analysis that compared the data for the simple distance task and the trials in the complex task on which nonfocal distance was 0 . In general, performance was better in the simple task. When the focal distance was 2 , however, performance was better in the complex task. This is what would have been expected if the participants had been using a two-dimensional decision rule in the complex task. In the simple task, the smallest distance on the negative trials was 3 , and the largest distance on the positive trials was 2 . Thus, the decision boundary would have been a critical value, lying between 3 and 2 . In the complex task, with the twodimensional rule, the smallest distance on the negative trials was again 3 , and the largest distance on the positive trials was in the interval from 2 to 2.83 . The decision boundary would have been all of the points whose aggregate distance was equal to a critical value, lying between 3 and a number in the interval from 2 to 2.83 (see Figure 4). We would have expected the critical value to be closer to 2 in the simple task than in the complex task. On simple task trials for which the focal distance was 2, the distance between the test circles was 2 . On complex task trials for which the focal distance was 2 and the nonfocal distance was 0 , the distance between the test circles was also 2. Because the distance between the circles would have been 2 in both cases, whereas the critical value would have been closer to 2 in the simple case, we would have expected poorer performance in the simple case.

Performance on the positive trials in the complex distance task depended as much on nonfocal as on focal distance. Both error rate and RT demonstrated a nominally but nonsignificantly stronger relationship with nonfocal than with focal distance. These results imply that nonfocal distance was given as much weight as focal distance in the aggregate distance on which response decisions were based. This, in turn, supports the early holistic over the dimensional similarity hypothesis. The required discrimination was easier to perform the greater the weight given to focal, as opposed to nonfocal, dis-

Table 4

Experiment 1: Performance in the Simple Distance Task and in Comparable Trials of the Complex Distance Task as a Function of Distance Between Test Circles on the Focal Dimension

\begin{tabular}{|c|c|c|c|c|c|c|c|c|c|c|c|c|}
\hline \multirow[b]{3}{*}{ Task } & \multicolumn{12}{|c|}{ Focal Distance (in Position Units) } \\
\hline & \multicolumn{2}{|c|}{1} & \multicolumn{2}{|c|}{2} & \multicolumn{2}{|c|}{3} & \multicolumn{2}{|c|}{4} & \multicolumn{2}{|c|}{5} & \multicolumn{2}{|c|}{6} \\
\hline & $M$ & $S E M$ & $M$ & SEM & $M$ & $S E M$ & $M$ & $S E M$ & $M$ & $S E M$ & $M$ & $S E M$ \\
\hline \multicolumn{13}{|c|}{ Error Rate } \\
\hline Complex & .036 & .010 & .042 & .013 & .646 & .045 & .220 & .041 & .141 & .049 & .070 & .026 \\
\hline Simple & .037 & .015 & .108 & .021 & .385 & .040 & .165 & .052 & .098 & .039 & .039 & .026 \\
\hline \multicolumn{13}{|c|}{ Response Time } \\
\hline Complex & 500 & 7 & 516 & 8 & 632 & 23 & 598 & 21 & 584 & 23 & 548 & 13 \\
\hline Simple & 506 & 9 & 542 & 14 & 576 & 22 & 559 & 19 & 545 & 15 & 546 & 21 \\
\hline
\end{tabular}

Note-Response time means include the $400 \mathrm{msec}$ that elapsed before the response signal appeared. 
tance. It would, therefore, have been advantageous to give more weight to focal, as opposed to nonfocal, distance. Such a weighting scheme should have been possible according to the dimensional similarity hypothesis, because access of information from interacting dimensions is always somewhat selective. Such a weighting scheme might not have been possible according to the early holistic hypothesis, because access of information from interacting dimensions can be completely unselective during the early stages of processing. Thus, the equivalent weighting that these results imply supports the early holistic over the dimensional similarity hypothesis. Note that the equivalent weighting of focal distance and nonfocal distance cannot have been induced by decision factors, inasmuch as the required discrimination was easier if focal distance was given greater weight than nonfocal distance was.

Of course, a number of alternative reasons might be advanced as to why performance on the positive trials in the complex distance task depended as much on nonfocal as on focal distance. One possibility is that the participants were insensitive to the decision contingencies of the task. They simply did not register that the discrimination could be performed more easily when focal distance was given greater weight than nonfocal distance was. Another possibility is that the participants were incapable of giving focal distance greater weight than nonfocal distance. Although differential weighting schemes have been implicated in the performance of other cognitive tasks (Nosofsky, 1986, 1987), such schemes may not have been viable in the present case. A third possibility is that the participants did give focal distance greater weight than they did nonfocal distance but that this was hidden, as a consequence of the fact that nonfocal distance was more salient than focal distance. A fourth possibility is that the difference between the effects of focal and nonfocal distance was so small that Experiment 1 was insensitive to it. This possibility is viable, inasmuch as the dimensional similarity hypothesis does not say exactly how much more heavily focal distance should have been weighted.

These alternative accounts might be called into question, and support for the early holistic hypothesis might be enhanced, if a greater effect could be shown for focal than for nonfocal distance, under conditions that matched the conditions in Experiment 1, except that the early holistic hypothesis predicted selective access of dimensional information. The early holistic hypothesis predicts this during the later stages of perceptual processing. The aim of Experiments $2 \mathrm{~A}$ and $2 \mathrm{~B}$, therefore, was to show a greater effect for focal than for nonfocal distance on positive trials in a version of the complex distance task that tapped later-stage processing.

Without testing systematically, it appears that performance on the negative trials in the complex distance task depended more on focal than on nonfocal distance. How can we account for the fact that focal distance and nonfocal distance had unequal effects on negative trials and equivalent effects on positive trials? First, we must note a simple fact of geometry: If the two points in a pair differ on two dimensions, $\mathrm{A}$ and $\mathrm{B}$, and the distance between the points is greater on Dimension A than on Dimension B, the Euclidean distance between the points will be increased to a greater degree by an increase in the interpoint distance on Dimension A than by an equivalent increase on Dimension B. Second, we must note that, on the negative trials, the distance between the test circles was always greater on the focal than on the nonfocal dimension. It follows that, for a given pair of negative test circles, an increase in the intercircle distance on the focal dimension increased the Euclidean distance between the circles to a greater degree than an equivalent increase on the nonfocal dimension did. Thus, if the participants were basing their responses on the Euclidean distance between the test circles, we would have expected greater focal than nonfocal distance effects on the negative trials, even with focal and nonfocal distance given equal weight.

In general, performance was better in the simple than in the complex distance task. By implication, the participants paid a cost for attending to two dimensions, rather than to one. This result will be discussed further below.

\section{EXPERIMENTS 2A AND 2B}

The aim of Experiments $2 \mathrm{~A}$ and $2 \mathrm{~B}$ was to show a greater effect of focal than of nonfocal distance on positive trials in a version of the complex distance task that tapped later-stage perceptual processing. The key difference between these experiments and Experiment 1 was in the temporal parameters. Specifically, the temporal interval between the presentation of the test circles and the response signal was greater in Experiments $2 \mathrm{~A}$ and $2 \mathrm{~B}$ than in Experiment 1. Given this difference, it was assumed that Experiments $2 \mathrm{~A}$ and $2 \mathrm{~B}$ would tap a later stage of processing than Experiment 1 did.

As in Experiment 1, it was expected that the twodimensional decision rule would be used in performing the complex distance task. The question at issue was the relative degree to which focal distance and nonfocal distance would be weighted in the aggregate distance on which response decisions were based. Both the early holistic and the dimensional similarity hypotheses predicted that focal distance would be given greater weight than nonfocal distance would be. These predictions followed from two premises. First, the task would be capable of being performed more easily to the extent that focal distance was given greater weight than nonfocal distance was, for the reasons given earlier. Second, access of dimensional information would be at least somewhat selective. In the case of the early holistic hypothesis, selectivity was predicted because the task would involve later-stage processing. In the case of the dimensional similarity hypothesis, selectivity was predicted because pairs of interacting dimensions are always differentiable, on the basis of attribute-level processing. It 
was reasoned that if focal distance was given greater weight than nonfocal distance was, a greater effect of focal than of nonfocal distance would be observed.

It was reasoned that such a result would enhance the support already provided for the early holistic hypothesis, in two senses. First, the result would directly confirm the predictions of the hypothesis. The support that would thereby be obtained for the hypothesis would be somewhat mitigated by virtue of the fact that the dimensional similarity hypothesis predicted the same result.

Second and more important, the result would confirm the support that Experiment 1 provided for the early holistic hypothesis. In that experiment, focal and nonfocal distance effects of equivalent size were observed in a task that could be performed more easily when focal distance was given greater weight than nonfocal distance was. This pattern of results was interpreted as an indication that access to dimensional information was completely unselective in that situation. This, in turn, was taken as support for the early holistic hypothesis. Several other possible interpretations of the effect were noted, however. According to one interpretation, the participants were insensitive to the fact that the task could be performed more easily when focal distance was given greater weight than nonfocal distance was. According to another interpretation, the participants were incapable of giving greater weight to focal distance than to nonfocal distance. According to another interpretation, the participants gave greater weight to focal distance than to nonfocal distance, but this was hidden as a consequence of the fact that nonfocal distance was more salient than focal distance. According to yet another interpretation, the difference between the effects of focal and nonfocal distance was too small to be detected in Experiment 1. Showing a greater effect of focal distance than of nonfocal distance in a task that was essentially identical to the task in Experiment 1 would render these alternative interpretations much less plausible.

\section{Experiment 2A}

Experiment $2 \mathrm{~A}$ was an initial attempt at showing a greater effect of focal than of nonfocal distance on positive trials in a complex distance task that tapped laterstage processing. The experiment was identical to Experiment 1, except in two respects: (1) The interval between the presentation of the test circles and the response signal was greater than that in Experiment 1, and (2) the reward contingencies were set to reduce yes errors, rather than no errors, as in Experiment 1. The latter change was made to ensure a large enough number of no errors that differential effects of focal and nonfocal distance could be detected.

\footnotetext{
Method

Participants. The participants were 14 students at George Washington University. They received extra credit in a psychology course in exchange for their efforts.

Stimuli. The stimuli were the same as in Experiment 1.
}

Design. The design was the same as in Experiment 1.

Procedure. The procedure was the same as in Experiment 1, except for the following changes. (1) The asterisks appeared 1,000 msec, rather than $400 \mathrm{msec}$ after the circles appeared. (2) The participant was told that he/she would receive a $\$ 5$ bonus if he/she made fewer than 20 yes errors and fewer than 100 no errors. When the participant made a yes error, a large red message appeared to that effect. When the participant made a no error, a small black message appeared to that effect.

\section{Results}

Complex distance task. Table 5 presents the data for positive trials as a function of focal and nonfocal distance between the test circles. Error rate increased with increases in focal and nonfocal distance. A regression analysis was conducted to confirm the significance of these patterns. The 112 cases in the analysis were the error rates that the 14 individual participants accrued for the eight different combinations of focal and nonfocal distance. To estimate the variability due to participants, 13 dummy variables were included. In other respects, the analysis was identical to the analyses that were done in Experiment 1. Table 6 reports the results of the analysis. Both focal and nonfocal distance accounted for significant amounts of the variance in the error rate data. Focal distance had a stronger impact than nonfocal distance did; the coefficient for focal distance was significantly larger than the coefficient for nonfocal distance $[t(111)=$ 1.70, one-tailed].

The RT data for positive trials were also analyzed as a function of focal and nonfocal distance (see Tables 5 and 6). Focal and nonfocal distance both accounted for significant amounts of the variance in the RT data. The coefficients for focal and nonfocal distance did not differ $[t(111)=0.95]$.

Table 7 presents the data for negative trials as a function of focal and nonfocal distance. An ANOVA revealed that error rate decreased as a function of focal distance [linear trend, $\left.F(1,13)=15.33, M S_{\mathrm{e}}=0.019\right]$ and as a function of nonfocal distance [linear trend, $F(1,13)=$ $\left.12.71, M S_{\mathrm{e}}=0.009\right]$ and that the linear trends in the ef-

Table 5

Experiment 2A: Performance on the Positive Trials in the Complex Distance Task as a Function of Distance Between Test Circles on the Focal and Nonfocal Dimensions

\begin{tabular}{|c|c|c|c|c|c|c|}
\hline \multirow{3}{*}{$\begin{array}{c}\text { Focal Distance } \\
\text { (in Position Units) }\end{array}$} & \multicolumn{6}{|c|}{ Nonfocal Distance (in Position Units) } \\
\hline & \multicolumn{2}{|c|}{0} & \multicolumn{2}{|c|}{1} & \multicolumn{2}{|c|}{2} \\
\hline & $M$ & $\overline{S E M}$ & $M$ & $\overline{S E M}$ & $M$ & $S E M$ \\
\hline \multicolumn{7}{|c|}{ Error Rate } \\
\hline 0 & & & .041 & .016 & .030 & .010 \\
\hline 1 & .047 & .018 & .038 & .013 & .031 & .008 \\
\hline 2 & .066 & .015 & .099 & .026 & .276 & .053 \\
\hline \multicolumn{7}{|c|}{ Response Time } \\
\hline 0 & & & 1,153 & 18 & 1,161 & 19 \\
\hline 1 & 1,156 & 18 & 1,156 & 18 & 1,165 & 19 \\
\hline 2 & 1,170 & 17 & 1,165 & 17 & 1,200 & 19 \\
\hline
\end{tabular}

Note-Response time means include the $1,000 \mathrm{msec}$ that elapsed before the response signal appeared. 
Table 6

Experiment 2A: Regression Analysis of Performance on the Positive Trials in the Complex Distance Task as a Function of Distance Between Test Circles on the Focal and Nonfocal Dimensions

\begin{tabular}{lccccc}
\hline \multicolumn{7}{c}{ Distance } & Coefficient & $\begin{array}{c}\text { Standard } \\
\text { Error }\end{array}$ & $\begin{array}{c}\text { Standardized } \\
\text { Coefficient }\end{array}$ & $t$ Value & $p$ Value \\
\hline \multicolumn{5}{c}{ Error Rate } \\
Focal & 0.071 & 0.012 & 0.482 & 5.86 & $<.001$ \\
Nonfocal & 0.046 & 0.012 & 0.315 & 3.84 & $<.001$ \\
$\quad R^{2}=.386$ & \multicolumn{7}{c}{} \\
\multicolumn{7}{c}{ Response Time } \\
Focal & 13.69 & 2.99 & 0.161 & 4.57 & $<.001$ \\
Nonfocal & 10.17 & 2.99 & 0.120 & 3.39 & $<.001$ \\
$\quad R^{2}=.887$ & & & & & \\
\hline
\end{tabular}

fects of focal and nonfocal distance interacted in the error rate data $\left[F(1,35)=13.16, M S_{\mathrm{e}}=0.008\right]$, because the effect of nonfocal distance was stronger for smaller values of focal distance.

RT decreased as a function of focal distance [linear trend, $\left.F(1,13)=8.36, M S_{\mathrm{e}}=1,920\right]$ and as a function of nonfocal distance [linear trend, $F(1,13)=4.37, M S_{\mathrm{e}}=$ $1,107]$. The effects of focal and nonfocal distance did not interact in the RT $[F(4,52)<1]$.

Simple distance task. Table 8 presents the data from the simple distance task and the comparable trials in the complex distance task. The error rates for the simple task and the comparable trials in the complex task did not differ $\left[F(1,13)=2.52, M S_{\mathrm{e}}=0.020\right]$. The error rates for both tasks were linear $\left[F(1,13)=23.93, M S_{\mathrm{e}}=0.002\right]$ and quadratic $\left[F(1,13)=16.49, M S_{\mathrm{e}}=0.008\right]$ functions of focal distance. The effects of task type and focal distance interacted in the error rate data $[F(5,65)=7.62$, $\left.M S_{\mathrm{e}}=0.003\right]$. The basis of this interaction was the difference between the effects of task type for a focal distance of 3 and for other values of focal distance. Although the error rates for the simple and the complex tasks generally did not differ, for focal distance $=3$, the error rate for the simple task was smaller than the error rate for the complex task $\left[F(1,13)=11.23, M S_{\mathrm{e}}=0.014\right]$.

RT did not differ for the simple task and the comparable trials of the complex task $[F(1,13)<1]$. RT for both tasks was neither a linear $[F(1,13)<1]$ nor a quadratic $\left[F(1,13)=3.77, M S_{\mathrm{e}}=2,563\right]$ function of focal distance. The effects of task type and focal distance did not interact in the RT data $[F(5,65)<1]$.

\section{Experiment 2B}

Although Experiment 2A demonstrated a larger effect of focal than of nonfocal distance, the results were not particularly robust. Experiment $2 \mathrm{~B}$ was an attempt to replicate these results. The experiment was identical to Experiment 2A, except that the horizontal and the vertical distances between adjacent positions in the position array were greater. The rationale for this change was as follows. Because the distances between the circles would be greater in Experiment 2B than in Experiment 2A, the task would be easier in Experiment $2 \mathrm{~B}$ than in Experiment 2A. A later processing stage might therefore have been reached in Experiment 2B than in Experiment 2A at the point that the response signal was presented. If this was the case, Experiment $2 \mathrm{~B}$ might have a better chance than Experiment $2 \mathrm{~A}$ of showing differential effects of focal and nonfocal distance.

\section{Method}

Participants. The participants were 36 students at George Washington University. They received extra credit in a psychology course in exchange for their efforts.

Stimuli. The stimuli were the same as in Experiments 1 and 2A, except that the horizontal and the vertical distances between adjacent positions in the position array were $11 \mathrm{~mm}$, rather than $8 \mathrm{~mm}$.

Design. The design was the same as in Experiments 1 and 2A.

Procedure. The procedure was the same as in Experiment 2A.

\section{Results}

Complex distance task. Table 9 presents the data for positive trials as a function of focal and nonfocal distance between the test circles. Error rate increased with increases in focal and nonfocal distance. A regression analysis was conducted to confirm the significance of these patterns. The 288 cases in the analysis were the error rates that the 36 individual participants accrued for the eight different combinations of focal and nonfocal distance. To estimate the variability due to participants, 35 dummy variables were included. In other respects, the analysis was identical to the analyses that were done in Experiments 1 and $2 \mathrm{~A}$. Table 10 reports the results of the analysis. Both focal and nonfocal distance accounted for significant amounts of the variance in the error rate data. More crucially for the object of the experiment, focal distance had a stronger impact than nonfocal distance did; the coefficient for focal distance was larger than the coefficient for nonfocal distance $[t(287)=1.74$, one-tailed]

The RT data for positive trials were also analyzed as a function of focal and nonfocal distance (see Tables 9 and

Table 7

Experiment 2A: Performance on the Negative Trials in the Complex Distance Task as a Function of Distance Between Test Circles on the Focal and Nonfocal Dimensions

\begin{tabular}{|c|c|c|c|c|c|c|}
\hline \multirow{3}{*}{$\begin{array}{c}\text { Focal Distance } \\
\text { (in Position Units) }\end{array}$} & \multicolumn{6}{|c|}{ Nonfocal Distance (in Position Units) } \\
\hline & \multicolumn{2}{|c|}{0} & \multicolumn{2}{|c|}{1} & \multicolumn{2}{|c|}{2} \\
\hline & $M$ & $\overline{S E M}$ & $M$ & SEM & $M$ & $S E M$ \\
\hline \multicolumn{7}{|c|}{ Error Rate } \\
\hline 3 & .251 & .056 & .168 & .050 & .073 & .021 \\
\hline 4 & .058 & .020 & .048 & .025 & .021 & .012 \\
\hline 5 & .041 & .018 & .066 & .021 & .035 & .02 \\
\hline 6 & .032 & .023 & & & & \\
\hline \multicolumn{7}{|c|}{ Response Time } \\
\hline 3 & 1,194 & 16 & 1,175 & 16 & 1,178 & 15 \\
\hline 4 & 1,170 & 20 & 1,173 & 21 & 1,158 & 18 \\
\hline 5 & 1,161 & 17 & 1,159 & 18 & 1,144 & 18 \\
\hline 6 & 1,166 & 21 & & & & \\
\hline
\end{tabular}

Note-Response time means include the $1,000 \mathrm{msec}$ that elapsed before the response signal appeared. 
Table 8

Experiment 2A: Performance in the Simple Distance Task and in Comparable Trials of the Complex Distance Task as a Function of Distance Between Test Circles on the Focal Dimension

\begin{tabular}{|c|c|c|c|c|c|c|c|c|c|c|c|c|}
\hline \multirow[b]{3}{*}{ Task } & \multicolumn{12}{|c|}{ Focal Distance (in Position Units) } \\
\hline & \multicolumn{2}{|c|}{1} & \multicolumn{2}{|c|}{2} & \multicolumn{2}{|c|}{3} & \multicolumn{2}{|c|}{4} & \multicolumn{2}{|c|}{5} & \multicolumn{2}{|c|}{6} \\
\hline & $M$ & $\overline{S E M}$ & $M$ & $\overline{S E M}$ & $M$ & $\overline{S E M}$ & $M$ & $\overline{S E M}$ & $M$ & $\overline{S E M}$ & $M$ & SEM \\
\hline \multicolumn{13}{|c|}{ Error Rate } \\
\hline Complex & .047 & .018 & .066 & .015 & .251 & .056 & .058 & .020 & .041 & .018 & .032 & .023 \\
\hline Simple & .040 & .021 & .063 & .017 & .103 & .045 & .033 & .025 & .024 & .017 & .024 & .024 \\
\hline \multicolumn{13}{|c|}{ Response Time } \\
\hline Complex & 1,156 & 18 & 1,170 & 17 & 1,194 & 16 & 1,170 & 20 & 1,161 & 17 & 1,166 & 21 \\
\hline Simple & 1,158 & 22 & 1,192 & 26 & 1,182 & 23 & 1,180 & 21 & 1,157 & 22 & 1,167 & 24 \\
\hline
\end{tabular}

10). Both focal and nonfocal distance accounted for significant amounts of the variance in the RT data. The coefficients for focal and nonfocal distance did not differ $[t(287)=0.16]$.

Table 11 presents the data for negative trials as a function of focal and nonfocal distance. An ANOVA revealed that error rate decreased as a function of focal distance [linear trend, $\left.F(1,35)=50.33, M S_{\mathrm{e}}=0.013\right]$ and as a function of nonfocal distance, [linear trend, $F(1,35)=$ $\left.21.16, M S_{\mathrm{e}}=0.008\right]$ and that the linear trends in the effects of focal and nonfocal distance interacted in the error rate data $\left[F(1,35)=18.34, M S_{\mathrm{e}}=0.007\right]$, because the effect of nonfocal distance was stronger for smaller values of focal distance.

RT did not vary as a function of focal distance $\left[F(2,70)=2.83, M S_{\mathrm{e}}=1,277\right]$ or as a function of nonfocal distance $[F(2,70)<1]$. The effects of focal and nonfocal distance did not interact in the RT data $[F(4,140)=$ $\left.2.28, M S_{\mathrm{e}}=660\right]$.

Simple distance task. Table 12 presents the data for the simple distance task and the comparable trials in the complex distance task. The error rates for the simple task and the comparable trials in the complex task did not differ $[F(1,35)<1]$. The error rates for both tasks were linear $\left[F(1,35)=8.85, M S_{\mathrm{e}}=0.011\right]$ and quadratic $[F(1,35)=$ $\left.21.24, M S_{\mathrm{e}}=0.009\right]$ functions of focal distance. The effects of task type and focal distance interacted in the error rate data $\left[F(5,175)=3.59, M S_{\mathrm{e}}=0.006\right]$. The basis of this interaction was the difference between the effects of task type for focal distance $=3$ and for other values of focal distance. Although the error rates for the simple and the complex tasks generally did not differ, for focal distance $=3$, the error rate was greater for the complex than for the simple task $\left[F(1,35)=5.97, M S_{\mathrm{e}}=\right.$ 0.014].

RT did not differ for the simple task and the comparable trials in the complex distance task $[F(1,35)<1]$. RT for both tasks was neither a linear $\left[F(1,35)=1.64, M S_{\mathrm{e}}=\right.$ $1,139]$ nor a quadratic $[F(1,35)<1]$ function of focal distance. The effects of task type and focal distance did not interact in the RT data $[F(5,175)<1]$.

\section{Discussion of Experiments 2A and 2B}

The test circles were more difficult to identify as being 2 or fewer position units apart on the focal dimension the further the circles were from one another on the focal and nonfocal dimensions. The test circles were easier to identify as being more than 2 position units apart on the focal dimension the further the circles were from one another on the focal and nonfocal dimensions. By implication, the task was performed on the basis of the twodimensional decision rule.

Further support for this conclusion comes from the analysis that compared the data for the simple distance task and the trials in the complex task on which nonfocal distance was 0 . In general, performance in the two tasks did not differ. When focal distance was 3 , however, performance was better in the simple task. This is what would have been expected if the participants had been using a two-dimensional decision rule in the complex task. As was argued in the discussion of Experiment 1, the critical value for positive versus negative responses would have been somewhere between 3 and 2 in the simple task and somewhere between 3 and a number in the interval from 2 to 2.83 in the complex task. Thus, we

Table 9

Experiment 2B: Performance on the Positive Trials in the Complex Distance Task as a Function of Distance Between Test Circles on the Focal and Nonfocal Dimensions

\begin{tabular}{|c|c|c|c|c|c|c|}
\hline \multirow{3}{*}{$\begin{array}{c}\text { Focal Distance } \\
\text { (in Position Units) }\end{array}$} & \multicolumn{6}{|c|}{ Nonfocal Distance (in Position Units) } \\
\hline & \multicolumn{2}{|c|}{0} & \multicolumn{2}{|c|}{1} & \multicolumn{2}{|c|}{2} \\
\hline & $M$ & SEM & $M$ & $S E M$ & $M$ & SEM \\
\hline \multicolumn{7}{|c|}{ Error Rate } \\
\hline 0 & & & .029 & .008 & .044 & .013 \\
\hline 1 & .033 & .012 & .030 & .006 & .076 & .018 \\
\hline 2 & .083 & .016 & .116 & .016 & .358 & .042 \\
\hline \multicolumn{7}{|c|}{ Response Time } \\
\hline 0 & & & 1,149 & 8 & 1,164 & 7 \\
\hline 1 & 1,149 & 7 & 1,154 & 7 & 1,158 & 8 \\
\hline 2 & 1,159 & 8 & 1,166 & 8 & 1,174 & 9 \\
\hline
\end{tabular}

Note-Response time means include the $1,000 \mathrm{msec}$ that elapsed before the response signal appeared. 
Table 10

Experiment 2B: Regression Analysis of Performance on the Positive Trials in the Complex Distance Task as a Function of Distance Between Test Circles on the Focal and Nonfocal Dimensions

\begin{tabular}{lccccc}
\hline \multicolumn{7}{c}{ Distance } & Coefficient & $\begin{array}{c}\text { Standard } \\
\text { Error }\end{array}$ & $\begin{array}{c}\text { Standardized } \\
\text { Coefficient }\end{array}$ & $t$ Value & $p$ Value \\
\hline \multicolumn{7}{c}{ Error Rate } \\
Focal & 0.096 & 0.009 & 0.483 & 10.97 & $<.001$ \\
Nonfocal & 0.077 & 0.009 & 0.390 & 8.85 & $<.001$ \\
$\quad R^{2}=.541$ & \multicolumn{7}{c}{} \\
\multicolumn{7}{c}{ Response Time } \\
Focal & 8.97 & 1.84 & 0.105 & 4.88 & $<.001$ \\
Nonfocal & 9.34 & 1.84 & 0.109 & 5.08 & $<.001$ \\
$\quad R^{2}=.891$ & & & & \\
\hline
\end{tabular}

would have expected the critical value to be closer to 3 in the complex than in the simple task. On simple task trials for which focal distance was 3, the distance between the test circles would have been 3. On complex task trials for which focal distance was 3 and nonfocal distance was 0 , the aggregate distance between the test circles would also have been 3 . Because the distance between the test circles would have been 3 in both cases, whereas the critical value would have been closer to 3 in the complex case, we would have expected poorer performance in the complex case.

Of greater interest, the error rate for positive trials depended to a greater degree on focal than on nonfocal distance. By implication, focal distance was given greater weight than nonfocal distance was in the aggregate distance on which response decisions were based.

These results accord with the predictions of both the early holistic and the dimensional similarity hypotheses. According to both hypotheses, these results were to be expected because the experimental task could be performed more easily with focal distance playing a larger role than nonfocal distance and because access to dimensional information was at least somewhat selective.

On balance, though, these results support the early holistic over the dimensional similarity hypothesis, because they reinforce the support that Experiment 1 offered for the former hypothesis. In that experiment, equivalent focal and nonfocal distance effects were observed even though decision factors dictated that focal distance be given greater weight than nonfocal distance was. It was concluded that access to information from the focal and nonfocal dimensions was completely unselective and that this argued for the early holistic hypothesis. Several other possible interpretations of the results were noted, however. It was suggested (1) that the participants might have been insensitive to the fact that focal distance should be given greater weight than nonfocal distance was and (2) that the participants might have been incapable of giving focal distance greater weight than they gave nonfocal distance. From the fact that focal distance had a stronger effect than nonfocal distance did in the present experiments, we can infer that neither of these problems existed in these experiments. Given that the present experiments were quite similar to Experiment 1, we can infer that, most likely, neither of these problems existed in Experiment 1. The support that Experiment 1 offered for the early holistic hypothesis is thereby reinforced.

It was also suggested that the participants in Experiment 1 might have given focal distance greater weight than they gave nonfocal distance, as the dimensional similarity hypothesis predicts, but that this might not have been detected, either (1) because nonfocal distance was more salient than focal distance or (2) because the difference between the effects of focal and nonfocal distance was too small for Experiment 1 to detect. The stronger effect of focal than of nonfocal distance in the present experiments calls these accounts into question. According to the dimensional similarity hypothesis, the difference in weighting between focal and nonfocal distance should have been no greater in Experiments 2A and 2B than in Experiment 1. Thus, if the difference went undetected in Experiment 1, it should also have gone undetected in Experiments 2A and 2B.

In both experiments, focal distance was only a slightly better predictor of performance than nonfocal distance was. By implication, access of dimensional information only gradually becomes selective in the complex distance task.

On negative trials, performance improved as a function of focal and nonfocal distance, with the effect of focal distance being apparently stronger than the effect of nonfocal distance. Again, these results are not particularly diagnostic. By the argument detailed in the Discussion section in Experiment 1, this is what would have been expected if the participants had used a two-dimensional Euclidean decision rule, regardless of whether focal distance was given greater weight than nonfocal distance was.

Whereas performance was better on the simple than on the complex distance task in Experiment 1, performance on the two tasks did not differ in Experiments 2A and $2 \mathrm{~B}$. The key to this discrepancy is probably the longer interval that elapsed in Experiments 2A and 2B between

Table 11

Experiment 2B: Performance on the Negative Trials in the Complex Distance Task as a Function of Distance Between Test Circles on the Focal and Nonfocal Dimensions

\begin{tabular}{|c|c|c|c|c|c|c|}
\hline \multirow{3}{*}{$\begin{array}{l}\text { Focal Distance } \\
\text { (in Position Units) }\end{array}$} & \multicolumn{6}{|c|}{ Nonfocal Distance (in Position Units) } \\
\hline & \multicolumn{2}{|c|}{0} & \multicolumn{2}{|c|}{1} & \multicolumn{2}{|c|}{2} \\
\hline & $M$ & SEM & $M$ & SEM & $M$ & $S E M$ \\
\hline \multicolumn{7}{|c|}{ Error Rate } \\
\hline 3 & .180 & .028 & .144 & .023 & .044 & .011 \\
\hline 4 & .036 & .010 & .024 & .004 & .023 & .003 \\
\hline 5 & .022 & .011 & .016 & .007 & .004 & .004 \\
\hline 6 & .040 & .015 & & & & \\
\hline \multicolumn{7}{|c|}{ Response Time } \\
\hline 3 & 1,169 & 10 & 1,166 & 10 & 1,164 & 9 \\
\hline 4 & 1,152 & 8 & 1,154 & 8 & 1,167 & 9 \\
\hline 5 & 1,159 & 9 & 1,157 & 9 & 1,151 & 10 \\
\hline 6 & 1,160 & 10 & & & & \\
\hline
\end{tabular}

Note-Response time means include the $1,000 \mathrm{msec}$ that elapsed before the response signal appeared. 
Table 12

Experiment 2B: Performance in the Simple Distance Task and in Comparable Trials of the Complex Distance Task as a Function of Distance Between Test Circles on the Focal Dimension

\begin{tabular}{|c|c|c|c|c|c|c|c|c|c|c|c|c|}
\hline \multirow[b]{3}{*}{ Task } & \multicolumn{12}{|c|}{ Focal Distance (in Position Units) } \\
\hline & \multicolumn{2}{|c|}{1} & \multicolumn{2}{|c|}{2} & \multicolumn{2}{|c|}{3} & \multicolumn{2}{|c|}{4} & \multicolumn{2}{|c|}{5} & \multicolumn{2}{|c|}{6} \\
\hline & $M$ & $S E M$ & $M$ & $S E M$ & $M$ & SEM & $M$ & $S E M$ & $M$ & $S E M$ & $M$ & $S E M$ \\
\hline \multicolumn{13}{|c|}{ Error Rate } \\
\hline Complex & .033 & .012 & .083 & .016 & .180 & .028 & .036 & .010 & .022 & .011 & .040 & .015 \\
\hline Simple & .037 & .009 & .106 & .029 & .112 & .021 & .019 & .008 & .046 & .018 & .028 & .016 \\
\hline \multicolumn{13}{|c|}{ Response Time } \\
\hline Complex & 1,149 & 7 & 1,159 & 8 & 1,169 & 10 & 1,152 & 8 & 1,159 & 9 & 1,160 & 10 \\
\hline Simple & 1,152 & 9 & 1,163 & 10 & 1,161 & 10 & 1,155 & 10 & 1,149 & 10 & 1,171 & 13 \\
\hline
\end{tabular}

the presentation of the test circles and the response signal. The participants were under less speed stress in Experiments $2 \mathrm{~A}$ and $2 \mathrm{~B}$. The differences that showed up under speed stress in Experiment 1 did not show up under these more relaxed conditions.

One loose end remains. In Experiment 1, equivalent effects of focal and nonfocal distance were observed with a short response interval and payoff conditions that discouraged no errors. In Experiments $2 \mathrm{~A}$ and 2B, differential effects of focal and nonfocal distance were observed with a long response interval and payoff conditions that discouraged yes errors. Prudence dictates that equivalent effects of focal and nonfocal distance be confirmed with a short response interval and payoff conditions that discourage yes errors. Experiment 3 was an attempt to do this.

\section{EXPERIMENT 3}

\section{Method}

Participants. The participants were 20 students at George Washington University. They received extra credit in a psychology course in exchange for their efforts.

Stimuli. The stimuli were the same as in Experiments 1 and 2A. Design. The design was the same as in Experiments 1,2A, and 2B.

Procedure. The procedure was the same as in Experiments 2A and $2 \mathrm{~B}$, except that the interval between the presentation of the test circles and the response signal was $400 \mathrm{msec}$, as in Experiment 1.

\section{Results}

Complex distance task. Table 13 presents the data for positive trials as a function of focal and nonfocal distance between the test circles. Error rate increased with increases in focal and nonfocal distance. A regression analysis was conducted to confirm the significance of these patterns. The 160 cases in the analysis were the error rates that the 20 individual participants accrued for the eight different combinations of focal and nonfocal distance. To estimate the variability due to participants, 19 dummy variables were included. In other respects, the analysis was identical to the analyses that were done in Experiments 1, 2A, and 2B. Table 14 reports the results of the analysis. Both focal and nonfocal distance ac- counted for significant amounts of the variance in the error rate data. More crucially for the object of the experiment, focal and nonfocal distance were equally strong predictors of error rate; the coefficients for the two forms of distance did not differ $[t(159)=0.20]$.

The RT data for positive trials were also analyzed as a function of focal and nonfocal distance (see Tables 13 and 14). Both focal and nonfocal distance accounted for significant amounts of the variance in the RT data. The coefficients for focal and nonfocal distance did not differ $[t(159)=0.32]$.

Table 15 presents the data for negative trials as a function of focal and nonfocal distance. An ANOVA revealed that error rate decreased as a function of focal distance [linear trend, $\left.F(1,19)=29.14, M S_{\mathrm{e}}=0.041\right]$ and as a function of nonfocal distance [linear trend, $F(1,19)=$ $\left.13.10, M S_{\mathrm{e}}=0.021\right]$ and that the linear trends in the effects of focal and nonfocal distance interacted in the error rate data $\left[F(1,19)=15.34, M S_{\mathrm{e}}=0.018\right]$, because the effect of nonfocal distance was stronger for smaller values of focal distance.

RT decreased as a function of focal distance [linear trend, $\left.F(1,19)=5.46, M S_{\mathrm{e}}=5,699\right]$ but did not vary as

Table 13

Experiment 3: Performance on the Positive Trials in the Complex Distance Task as a Function of Distance Between Test Circles on the Focal and Nonfocal Dimensions

\begin{tabular}{|c|c|c|c|c|c|c|}
\hline \multirow{3}{*}{$\begin{array}{c}\text { Focal Distance } \\
\text { (in Position Units) }\end{array}$} & \multicolumn{6}{|c|}{ Nonfocal Distance (in Position Units) } \\
\hline & \multicolumn{2}{|c|}{0} & \multicolumn{2}{|c|}{1} & \multicolumn{2}{|c|}{2} \\
\hline & $M$ & $S E M$ & $M$ & SEM & $M$ & $S E M$ \\
\hline \multicolumn{7}{|c|}{ Error Rate } \\
\hline 0 & & & .132 & .045 & .170 & .045 \\
\hline 1 & .144 & .044 & .139 & .037 & .198 & .045 \\
\hline 2 & .168 & .047 & .202 & .049 & .447 & .042 \\
\hline \multicolumn{7}{|c|}{ Response Time } \\
\hline 0 & & & 514 & 12 & 550 & 13 \\
\hline 1 & 520 & 9 & 520 & 10 & 560 & 12 \\
\hline 2 & 538 & 11 & 551 & 13 & 599 & 26 \\
\hline
\end{tabular}

Note-Response time means include the $400 \mathrm{msec}$ that elapsed before the response signal appeared. 
Table 14

Experiment 3: Regression Analysis of Performance on the Positive Trials in the Complex Distance Task as a Function of Distance Between Test Circles on the Focal and Nonfocal Dimensions

\begin{tabular}{lccccc}
\hline \multicolumn{7}{c}{ Distance } & Coefficient & $\begin{array}{c}\text { Standard } \\
\text { Error }\end{array}$ & $\begin{array}{c}\text { Standardized } \\
\text { Coefficient }\end{array}$ & $t$ Value & $p$ Value \\
\hline \multicolumn{5}{c}{ Error Rate } \\
Focal & .083 & .011 & .305 & 7.47 & $<.001$ \\
Nonfocal & .081 & .011 & .295 & 7.24 & $<.001$ \\
$\quad R^{2}=.782$ & & & & & \\
& \multicolumn{7}{c}{ Response Time } \\
Focal & 22.55 & 4.38 & .264 & 5.13 & $<.001$ \\
Nonfocal & 27.33 & 4.38 & .322 & 6.24 & $<.001$ \\
$\quad R^{2}=.653$ & & & & & \\
\hline
\end{tabular}

a function of nonfocal distance $\left[F(2,38)=1.49, M S_{\mathrm{e}}=\right.$ $2,281]$. The effects of focal and nonfocal distance interacted in the RT data $\left[F(4,76)=4.19, M S_{\mathrm{e}}=1,229\right]$, because the effect of focal distance was larger for small than for large values of nonfocal distance.

Simple distance task. Table 16 presents the data for the simple distance task and the comparable trials in the complex distance task. The error rates for the simple task and the comparable trials in the complex task did not differ $[F(1,19)<1]$. The error rates for the two tasks were linear $\left[F(1,19)=7.82, M S_{\mathrm{e}}=0.023\right]$ and quadratic $\left[F(1,19)=23.18, M S_{\mathrm{e}}=0.044\right]$ functions of focal distance. The effects of task type and focal distance did not interact in the error rate data $\left[F(5,95)=1.62, M S_{\mathrm{e}}=\right.$ 0.023].

RT did not differ in the simple task and the comparable trials in the complex task $[F(1,19)<1]$. RT for both tasks was a linear $\left[F(1,19)=30.30, M S_{\mathrm{e}}=3,727\right]$, but not a quadratic, function of focal distance $[F(1,195)=$ $\left.3.13, M S_{\mathrm{e}}=5,828\right]$. The effects of task type and focal distance interacted in the $\mathrm{RT}$ data $\left[F(5,95)=2.50, M S_{\mathrm{e}}=\right.$ $2,102]$. The basis of this interaction was the difference between the effects of task type for focal distance $=3$ and for other values of focal distance. Although RTs for the simple and the complex tasks generally did not differ, at focal distance $=3$, RT was greater for the complex task than for the simple task $\left[F(1,19)=10.38, M S_{\mathrm{e}}=\right.$ $1,824]$.

\section{Discussion}

The basic pattern in Experiment 1 was replicated here. Most important, performance depended to the same degree on the focal and the nonfocal distance between the test circles. The most notable difference between the two sets of results emerges in the comparison between the data for the simple distance task and the comparable trials in the complex distance task. Two discrepancies are worthy of note. First, in Experiment 1, performance was significantly better in the simple than in the complex distance task. In Experiment 3, however, this was not the case. The pattern in Experiment 1 was nominally, but not significantly, present. Although its exact basis is unclear, this discrepancy may reflect the difference in payoff criteria between the two experiments. Second, in Experiment 1 , performance in the simple distance task was disproportionately bad for focal distance $=2$. In Experiment 3, however, performance in the complex distance task was disproportionately bad for focal distance $=3$. The reason for this discrepancy is again unclear. Recall, however, from the previous discussion, that the patterns of both experiments can be taken as evidence for the use of the two-dimensional decision rule. In that sense, then, the two sets of results cohere.

\section{GENERAL DISCUSSION}

The results of four experiments supported the early holistic hypothesis over the dimensional similarity hypothesis. The experiments explored perceptual processing with respect to a pair of interacting dimensions. The participants in each experiment performed a task that required them to evaluate stimuli on one of the two dimensions along which they varied. The task could be performed in two ways: (1) on the basis of information from the dimension that was focal to the judgment required and (2) on the basis of information from the nonfocal, as well as the focal, dimension. The task could be performed more easily to the extent that it was performed solely on the basis of focal information.

The task in Experiments 1 and 3 was designed to tap early stages of perceptual processing. Information from the focal and the nonfocal dimensions exerted equivalent effects on performance of the task. By implication, the task was performed on the basis of a decision rule that gave equal weight to focal and nonfocal information. One interpretation of this result is that equal weight was given because access to dimensional information was completely unselective. Such a lack of selectivity is consistent with the early holistic hypothesis and inconsistent with the dimensional similarity hypothesis. This inter-

Table 15

Experiment 3: Performance on the Negative Trials in the Complex Distance Task as a Function of Distance Between Test Circles on the Focal and Nonfocal Dimensions

\begin{tabular}{|c|c|c|c|c|c|c|}
\hline \multirow{3}{*}{$\begin{array}{c}\text { Focal Distance } \\
\text { (in Position Units) }\end{array}$} & \multicolumn{6}{|c|}{ Nonfocal Distance (in Position Units) } \\
\hline & \multicolumn{2}{|c|}{0} & \multicolumn{2}{|c|}{1} & \multicolumn{2}{|c|}{2} \\
\hline & $M$ & $\overline{S E M}$ & $M$ & $\overline{S E M}$ & $M$ & $S E M$ \\
\hline \multicolumn{7}{|c|}{ Error Rate } \\
\hline 3 & .441 & .052 & .421 & .049 & .194 & .045 \\
\hline 4 & .179 & .037 & .190 & .039 & .153 & .036 \\
\hline 5 & .161 & .048 & .151 & .047 & .149 & .048 \\
\hline 6 & .108 & .045 & & & & \\
\hline \multicolumn{7}{|c|}{ Response Time } \\
\hline 3 & 632 & 22 & 600 & 16 & 589 & 12 \\
\hline 4 & 577 & 16 & 569 & 12 & 590 & 18 \\
\hline 5 & 573 & 16 & 568 & 12 & 583 & 18 \\
\hline 6 & 563 & 22 & & & & \\
\hline
\end{tabular}

Note-Response time means include the $400 \mathrm{msec}$ that elapsed before the response signal appeared. 
Table 16

Experiment 3: Performance in the Simple Distance Task and Comparable Trials of the Complex Distance Task as a Function of Distance Between Test Circles on the Focal Dimension

\begin{tabular}{|c|c|c|c|c|c|c|c|c|c|c|c|c|}
\hline \multirow[b]{3}{*}{ Task } & \multicolumn{12}{|c|}{ Focal Distance (in Position Units) } \\
\hline & \multicolumn{2}{|c|}{1} & \multicolumn{2}{|c|}{2} & \multicolumn{2}{|c|}{3} & \multicolumn{2}{|c|}{4} & \multicolumn{2}{|c|}{5} & \multicolumn{2}{|c|}{6} \\
\hline & $M$ & $S E M$ & $M$ & $S E M$ & $M$ & $S E M$ & $M$ & $S E M$ & $M$ & SEM & $M$ & $S E M$ \\
\hline \multicolumn{13}{|c|}{ Error Rate } \\
\hline Complex & .144 & .044 & .168 & .047 & .441 & .052 & .179 & .037 & .161 & .048 & .108 & .045 \\
\hline Simple & .120 & .039 & .204 & .051 & .316 & .047 & .149 & .040 & .160 & .054 & .100 & .050 \\
\hline \multicolumn{13}{|c|}{ Response Time } \\
\hline Complex & 520 & 9 & 538 & 11 & 632 & 22 & 577 & 16 & 573 & 16 & 563 & 22 \\
\hline Simple & 516 & 12 & 561 & 20 & 589 & 24 & 568 & 17 & 551 & 16 & 544 & 23 \\
\hline
\end{tabular}

pretation is not conclusive, however, inasmuch as the results could have reflected other secondary factors, such as insensitivity to decision factors, incapacity for relative weighting of stimulus dimensions, the relative salience of focal and nonfocal distance, or the insensitivity of Experiments 1 and 3 to differences in the weighting of focal and nonfocal distance.

The task in Experiments 2A and 2B was designed to tap a later stage of perceptual processing than the task in Experiments 1 and 3 did. Focal information exerted a larger effect on performance of the task than nonfocal information did. By implication, the task was performed on the basis of a decision rule that gave greater weight to focal than to nonfocal information. Inasmuch as the secondary factors mentioned earlier should have been present in Experiments 2A and 2B, as well as Experiments 1 and 3, the results of Experiments $2 \mathrm{~A}$ and $2 \mathrm{~B}$ suggest that the results of Experiments 1 and 3 do not reflect such factors. Taken together, the results of the four experiments support the early holistic hypothesis over the dimensional similarity hypothesis.

Note that the task in the present study bears some resemblance to both the correlated and the orthogonal values tasks. The task resembles the correlated values task in that nonfocal, as well as focal, information is used in performing it. Note, however, that, whereas the use of nonfocal information may be induced by decision factors in the correlated values task, this is not the case in the present task, because the required discrimination can be performed more easily to the extent that nonfocal information is excluded. The task resembles the orthogonal values task in the absence of a useful correlation between the values on the focal and the nonfocal dimensions (actually, a small negative correlation [-.231] exists between values on the two dimensions, as a consequence of the fact that no pair of circles exists with zero distance on the focal and the nonfocal dimensions; this correlation cannot be used to guide responding, though, in contrast to the case of the correlated values task). Note, however, that, whereas nonfocal information is irrelevant to performance of the orthogonal values task, nonfocal in- formation is relevant to the performance of the present task. The task can be performed most easily if nonfocal information is excluded, but it can be successfully performed on the basis of an aggregation of focal and nonfocal information.

Support for the early holistic hypothesis appeared here in a somewhat different guise than in past work. Functional relationships were demonstrated between dimensional information and measures of performance. Functional relationships such as these have not typically been demonstrated in past work. One common strategy has been to measure the size of the redundancy and/or the interference effect, aggregated across stimulus values on the focal and nonfocal dimensions, as a function of variables of interest (Melara \& Day, 1992; Melara \& Marks, 1990a, 1990b; Melara et al., 1993b; Melara \& Mounts, 1994; Mounts \& Melara, 1995). Another common strategy has been to compare performance on stimulus items that have congruent and incongruent values on two stimulus dimensions, where each of the dimensions in question has only two values (Melara \& Marks, 1990b; Melara et al., 1993b). (In one case, the stimulus dimensions had more than two values, but here the comparison of the congruent and the incongruent cases involved a complex estimation process; Melara \& Mounts, 1994.)

The nearest thing, in past work, to the functional relationships of the present study has been observed in tasks that combine elements of the correlated and the orthogonal values tasks (Melara \& Marks, 1990a; Mounts \& Melara, 1995). These tasks have suffered, however, from the same flaw that complicates interpretation of the simple version of the correlated values task - that is, the use of nonfocal information may have been induced by the decision rule. The present task does not suffer from this flaw.

It may be necessary to explore functional relationships such as those demonstrated here in order to choose between the early holistic and the dimensional similarity hypotheses. Both hypotheses can accommodate the impact of nonfocal information on perceptual judgments. The hypotheses differ only in the degree of impact that 
they allow. Whereas the early holistic hypothesis allows nonfocal information to have as much impact as focal information does, the dimensional similarity hypothesis does not. Thus, in order to choose between the hypotheses, it may be necessary to quantify the impact of focal and nonfocal information in the manner of the present study.

Although the present results support the early holistic hypothesis over the dimensional similarity hypothesis, these results may require that we reformulate the former hypothesis. How might we accommodate the present results under that hypothesis? Access to dimensional information was at least somewhat selective in Experiments $2 \mathrm{~A}$ and $2 \mathrm{~B}$. Thus, we would have to say that the task of these experiments was performed on the basis of a two-dimensional mental representation. Consider the following as an example of such a representation. A given pair of test circles are represented as points in a space whose dimensions are scaled to reflect a relative weighting of focal and nonfocal distance. More specifically, the nonfocal dimension of this space is shrunken, relative to the focal dimension (see Figure 4B). The response decision is based on the Euclidean distance between the points. If this distance is smaller than the critical value, a positive response is emitted. Note that the focal and the nonfocal dimensions are differentiated in terms of scale in this representation

Representations such as this have been proposed in previous work. It has been suggested that the dimensions of cognitive space expand and contract with their importance for the judgment that is required in a given context. Thus, a dimension that is unimportant for the judgment shrinks, relative to a dimension that is important for the judgment (Maddox, 1992; Nosofsky, 1986, 1987).

What kind of representation was used, then, in Experiments 1 and 3? Access to dimensional information was completely unselective here. Traditional forms of the early holistic hypothesis would hold that the task was performed on the basis of a one-dimensional mental representation. In such a representation, a given pair of test circles would be represented as a single point, corresponding to the Euclidean distance that separated them on the computer screen. If this distance was smaller than the critical value, a positive response would be emitted.

Taken in conjunction with our account of Experiments $2 \mathrm{~A}$ and $2 \mathrm{~B}$, this account raises problems, however. Specifically, it is unclear how we get from the one-dimensional representation of Experiments 1 and 3 to the twodimensional representation of Experiments 2A and 2B. The former representation is in use $500 \mathrm{msec}$ after the presentation of the test circles. The latter representation is in use $600 \mathrm{msec}$ later. How is the latter representation derived from the former in $600 \mathrm{msec}$ ? One possible response to this dilemma involves rethinking the representation for Experiments 1 and 3. Instead of a one-dimensional representation, we might consider a two-dimensional representation in which the two dimensions are undifferentiated in terms of scale.
Thus, building on ideas suggested by Maddox (1992), we might reformulate the early holistic hypothesis as follows, for the case of distance judgments with respect to the horizontal and vertical dimensions of physical space, and possibly for other perceptual judgments and other pairs of interacting dimensions. Horizontal and vertical position are always differentiated in representational terms, even in the early stages of perceptual processing that is, they are always represented in terms of a twodimensional mental space. In terms of the cognitive processing required for judgments of distance, however, the two dimensions are undifferentiated in early processing, because participants can only access the aggregate distance between a pair of stimuli. When selective access is required of the distance on one dimension, attention is focused on the dimension in question, and the other dimension is ignored. The ultimate impact of focusing attention one of the two dimensions is that the nonfocal dimension shrinks to the point that its values are indistinguishable. It is not clear by what mechanism the shrinkage is accomplished, although an inhibitory process of some sort is probably involved (see, e.g., Melara \& Algom, 2003). In any case, once the shrinkage has occurred, differences on the nonfocal dimension have no impact on the evaluation process. Shrinkage of the nonfocal dimension is a gradual process, however. During the early stages of perceptual processing, the nonfocal dimension does not shrink at all. Thus, even though the objective is to evaluate a stimulus on one of the two dimensions, the stimulus is of necessity evaluated on both dimensions.

This formulation of the early holistic hypothesis conceives dimensional differentiation differently than previous formulations do. In previous formulations, two interacting dimensions were represented as two mental dimensions if access to dimensional information was selective and as one mental dimension if it was not. In the present formulation, two interacting dimensions are always represented as two mental dimensions. Those dimensions are differentiated in terms of scale if access to dimensional information is selective but are undifferentiated in terms of scale if access is unselective.

The present formulation of the early holistic hypothesis is more similar than previous formulations to the dimensional similarity hypothesis. Most important, the present formulation holds, in common with the dimensional similarity hypothesis, that two interacting dimensions are always represented as two mental dimensions, even in the early stages of perceptual processing. In contrast to the dimensional similarity hypothesis, however, the present formulation holds that access to information concerning two interacting dimensions is unselective during the early stages of processing.

The present formulation of the early holistic hypothesis receives some support from the results of the comparison that was done, in Experiment 1, between performance in the simple distance task and that for the comparable trials in the complex distance task. In both cases, the vertical 
distance between the test circles was 0 and the horizontal distance varied from 1 to 6 . Yet performance was better in the simple distance task. On the assumption that this result is reliable (and that the failure to replicate it in Experiment 3 has some reasonable explanation), one possible explanation for the result is that the test circles were represented in a two-dimensional mental space for the complex task and in a one-dimensional space for the simple distance task. There are, of course, other possible explanations for the result. For example, the complex task required that the participants attend to more spatial locations than the simple task did. Nonetheless, the result is worth exploring further.

Of course, the conclusions above come with several caveats. The largest concern is that, although the conclusions pertain to processes of early visual perception, such processes were not directly observed in the present study. The study was set up to allow inferences about perceptual processes on the basis of patterns of decision rule usage across several experiments. The inferences that were drawn seem fair and defensible. One would prefer to see the inferences reinforced, however, with results that more directly reflect perceptual processes. This will require a more complex, model-based approach. Work is underway toward this end.

A secondary reservation concerns the response signal procedure that was used to control temporal factors in the different experiments. This procedure was necessary, in that the crucial predictions of the early holistic hypothesis concern the early stages of perceptual processing. In order to test these predictions, it was necessary to tap those early processing stages. The response signal procedure is generally accepted as a means of doing this. Nonetheless, it is possible that the procedure encouraged unnatural response tendencies that corrupted the results. One would prefer to see the results reinforced using other, less intrusive procedures.

Finally, it must be noted that the present results reflect data from only one pair of dimensions. This does not detract from the support offered for the early holistic hypothesis. The dimensional similarity hypothesis holds that access to information from pairs of interacting dimensions is always somewhat selective during the early stages of processing. Thus, the demonstration that access to information from the two dimensions in Experiments 1 and 3 was unselective is sufficient to make the crucial theoretical point.

It seems clear, nonetheless, that the generality of the present results must be explored in further work. Certainly, the results are unlikely to generalize to all pairs of interacting dimensions. It was crucial to the results observed here that pairs of distances on the two dimensions of the stimulus set were capable of being aggregated to form single distances. In order that this be possible for a given pair of dimensions, each of the dimensions in the pair must have a polar structure, such that values at the two ends of each of the dimensions are maximally dif- ferent from one another. In addition, in order for the present phenomenon to be studied with a particular pair of dimensions, the two dimensions must be capable of unambiguous alignment. In other words, all of the participants must agree all of the time as to which end of each dimension holds the low values.

In conclusion, the present results suggest that access of horizontal and vertical position information is unselective during the early stages of perceptual processing but becomes selective during the later stages of processing. These results support the early holistic hypothesis over the dimensional similarity hypothesis.

\section{REFERENCES}

GARNER, W. R. (1974). The processing of information and structure. Hillsdale, NJ: Erlbaum.

Garner, W. R., \& Felfoldy, G. L. (1970). Integrality of stimulus dimensions in various types of information processing. Cognitive Psychology, 1, 225-241.

Grau, J. W., \& Kemler Nelson, D. G. (1988). The distinction between integral and separable dimensions: Evidence for the integrality of pitch and loudness. Journal of Experimental Psychology: General, 117, 347-370.

Kemler Nelson, D. G. (1993). Processing integral dimensions: The whole view. Journal of Experimental Psychology: Human Perception \& Performance, 19, 1105-1113.

Lockhead, G. R. (1972). Processing dimensional stimuli: A note. Psychological Review, 79, 410-419.

LockHEAD, G. R. (1979). Holistic versus analytic process models: A reply. Journal of Experimental Psychology: Human Perception \& Performance, 5, 746-755.

MadDox, W. T. (1992). Perceptual and decisional separability. In F. G. Ashby (Ed.), Multidimensional models of perception and cognition (pp. 147-180). Hillsdale, NJ: Erlbaum.

MadDox, W. T. (2001). Separating perceptual processes from decisional processes in identification and categorization. Perception \& Psychophysics, 63, 1183-1200.

Maddox, W. T., \& Ashby, F. G. (1996). Perceptual separability, decisional separability, and the identification-speed classification relationship. Journal of Experimental Psychology: Human Perception \& Performance, 22, 795-817.

Melara, R. D. (1989). Dimensional interaction between color and pitch. Journal of Experimental Psychology: Human Perception \& Performance, 15, 69-79.

Melara, R. D. (1992). The concept of perceptual similarity: From psychophysics to cognitive psychology. In D. Algom (Ed.), Psychophysical approaches to cognition (pp. 303-388). Amsterdam: Elsevier, North-Holland.

Melara, R. D., \& Algom, D. (2003). Driven by information: A tectonic theory of Stroop effects. Psychological Review, 110, 422-471.

Melara, R. D., \& Day, D. J. A. (1992). Primacy of dimensions in vibrotactile perception: An evaluation of early holistic models. Perception \& Psychophysics, 52, 1-17.

Melara, R. D., \& Marks, L. E. (1990a). HARD and sofT interacting dimensions: Differential effects of dual context on classification. Perception \& Psychophysics, 47, 307-325.

Melara, R. D., \& Marks, L. E. (1990b). Perceptual primacy of dimensions: Support for a model of dimensional interaction. Journal of Experimental Psychology: Human Perception \& Performance, 16, 398414.

Melara, R. D., Marks, L. E., \& Potts, B. C. (1993a). Early-holistic processing or dimensional similarity? Journal of Experimental Psychology: Human Perception \& Performance, 19, 1114-1120.

Melara, R. D., Marks, L. E., \& Potts, B. C. (1993b). Primacy of dimensions in color perception. Journal of Experimental Psychology: Human Perception \& Performance, 19, 1082-1104. 
Melara, R. D., \& Mounts, J. R. W. (1994). Contextual influences on interactive processing: Effects of discriminability, quantity, and uncertainty. Perception \& Psychophysics, 56, 73-90.

Mounts, J. R. W., \& Melara, R. D. (1995). Classification of color dimensions in multiple contexts. Journal of Experimental Psychology: Human Perception \& Performance, 21, 257-274.

NosofsKy, R. M. (1986). Attention, similarity, and the identificationcategorization relationship. Journal of Experimental Psychology: General, 115, 39-57.
Nosofsky, R. M. (1987). Attention and learning processes in the identification and categorization of integral stimuli. Journal of Experimental Psychology: Learning, Memory, \& Cognition, 13, 87-109.

SMITH, L. B., \& KeMLER, D. G. (1978). Levels of experienced dimensionality in children and adults. Cognitive Psychology, 10, 502-532.

(Manuscript received July 24, 2003;

revision accepted for publication July 20, 2004.) 